\title{
Stellar populations in the center of barred spiral galaxies
}

\author{
M. Mollá ${ }^{1}$, S. Cantin ${ }^{2}$, C. Robert ${ }^{2}$ and A. Pellerin ${ }^{3}$ \\ ${ }^{1}$ Depto. de Investigación Básica, CIEMAT Avda. Complutense 22, 28040 Madrid, Spain $\dagger$ \\ ${ }^{2}$ Université Laval, Dept. de physique, de génie physique et d'optique, and Observatoire du \\ mont Mégantic, Québec, QC G1K 7P4, Canada \\ ${ }^{3}$ Space Telescope Science Institute, 3700 San Martin Drive, Baltimore MD 21218, U.S.A.
}

\begin{abstract}
We show observations obtained with the integral field spectrometer OASIS for the centers of a sample of barred galaxies. The high spatial resolution of the instrument allows to distinguish spatially some structures within these regions as defined by stellar populations of different ages and metallicities. From these data we deduce important clues about the star formation history. But we advise that, in order to obtain adequately the evolutionary sequence, a combination of chemical and synthesis models might be necessary.
\end{abstract}

Keywords. galaxies: stellar content, galaxies: bulges, galaxies: spirals

\section{Observing disk galaxies}

When observing galaxies, we may obtain information about different evolutionary times. On one hand we may get emission lines coming from the gas, which characterize the present state of a galaxy and provide the nebular elemental abundances, and/or the star formation rate of the last bursts. They are usually used as constraints for chemical evolution models. On the other hand, we may get brightness, colors and/or spectral absorption indices, all of them stellar populations indicators giving information about the properties averaged along the whole time of evolution, and which are interpreted by means of synthesis models. In this work we analyze a set of data obtained for the central regions of a sample of barred galaxies. There are gas and stars in these regions and, therefore, we will try to constraint the possible histories using both types of information.

Our observations were taken in the Canada-France-Hawai Telescope in 2001. We used the instrument OASIS, a microlens matrix imaging spectrometer with two configurations: in the blue, with a wavelength range from 4700 to $5500 \AA$, and in the red, from 6200 to $6850 \AA$. Its spatial resolution is $0.42^{\prime \prime}$ by lenslet and the spectral resolution is $\sim 2 \AA /$ pix. In this piece of work we show the preliminary results corresponding to 4 barred galaxies: NGC 2718 (SABab), NGC 4385 (SB0+), NGC 4900 (SBc) and NGC 5430 (SBb).

The data were reduced with the XOASIS package (version 6.0), following the classical steps: inversion of images, elimination of the over-scan, subtraction of dark current, subtraction of the bias, mask extraction and wavelength calibration. This way we got a data-cube with $\sim 800$ spectra by galaxy. Then, we did the flat field correction, eliminated the cosmic rays and subtracted the sky background. Finally, we performed a flux calibration and corrected the spectra of the Galactic extinction with a Seaton law, using a $E(B-V)=0.024$. After to put the spectra in their rest frame, we reduced our spatial resolution from 0.41 arcsec to 0.8 arcsec, our seeing limit.

$\dagger$ email: mercedes.molla@ciemat.es 

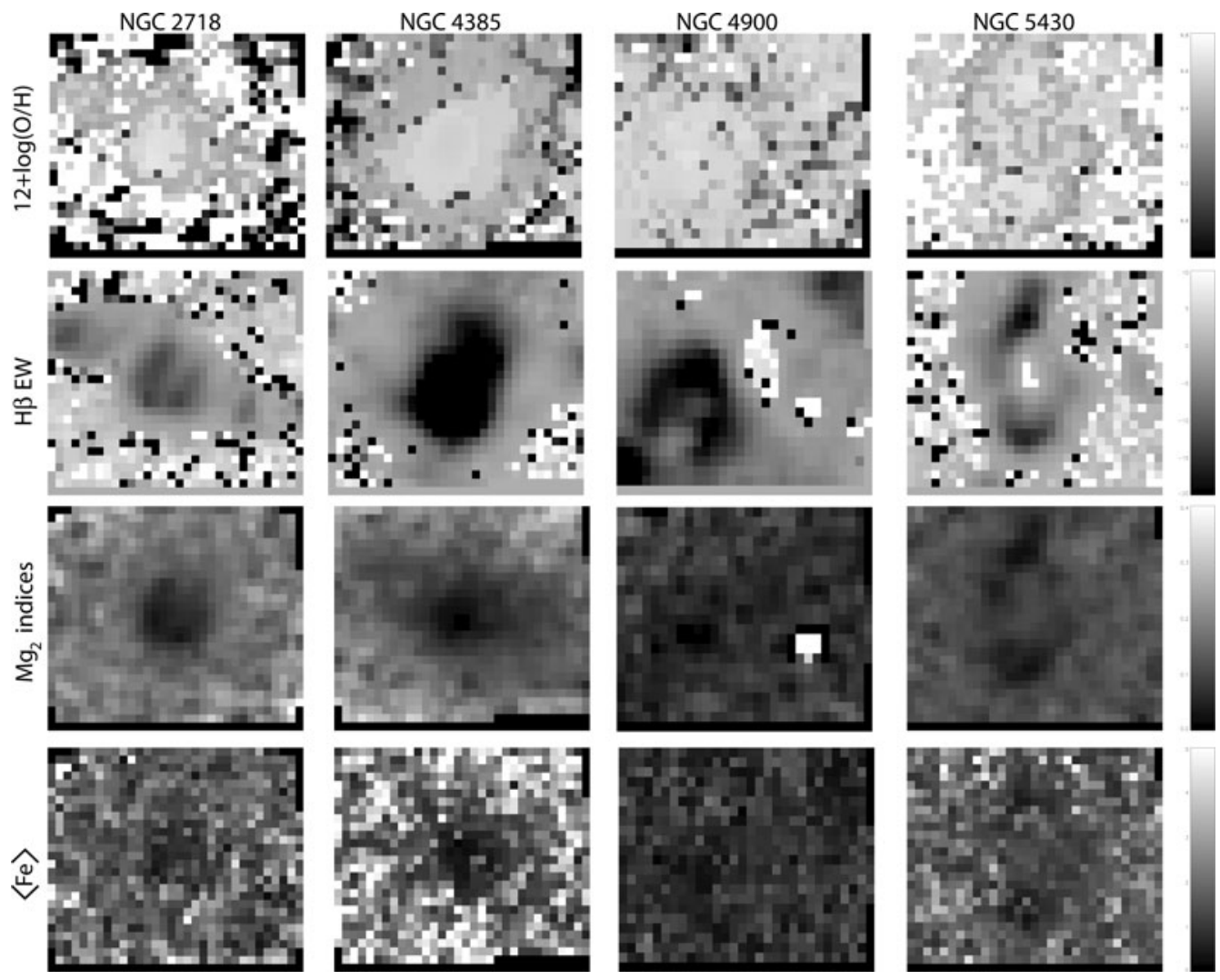

Figure 1. Maps shown in rows: 1) oxygen abundance; 2) EW $\mathrm{H} \beta(\AA) ; 3)$ the index $\mathrm{Mg} 2$ (mag); 4) the index $<\mathrm{Fe}>(\AA)$. Each column for a galaxy as labeled.

After to perform the previously described steps, we measured the emission line intensities and we estimate the internal extinction, which is in a range between 0 to $3.6 \mathrm{mag}$, with a mean value of $\langle\mathrm{E}(\mathrm{B}-\mathrm{V})\rangle=0.33$, from $\mathrm{H} \alpha$ and $\mathrm{H} \beta$. The spectra are corrected using the reddening law of Cardelli et al. (1989).

\section{Results}

Oxygen abundances were estimated from the emission line ratios following the method by Kewley \& Dopita (2002). The absorption spectral indices, Mg2,Fe5270 and Fe5335 and $\mathrm{H} \beta$, were calculated within IRAF by fitting a Gaussian around the strongest feature near the theoretical line wavelength value.

We show our results in Fig. 1. In the first row, we see that oxygen abundances are very uniform within each galaxy, with small differences between galaxies. In the second row, we show the map of $\mathrm{EW}(\mathrm{H} \beta)$ with positive and negative values, corresponding to absorption and emission lines. It is clear that the 4 galaxies show one (or several) region(s) where $\mathrm{H} \beta$ in emission is intense (dark regions), which correspond to the strongest recent star-bursts. In NGC 2718 and NGC 4385 it occurs in the whole central region, elongated in NGC 2718, around 10 arcsec wide, while NGC 5430 shows some circumnuclear HII regions around the center. In NGC 4900 the emission of $\mathrm{H} \beta$ occurs in an region at the south-east. 
Age
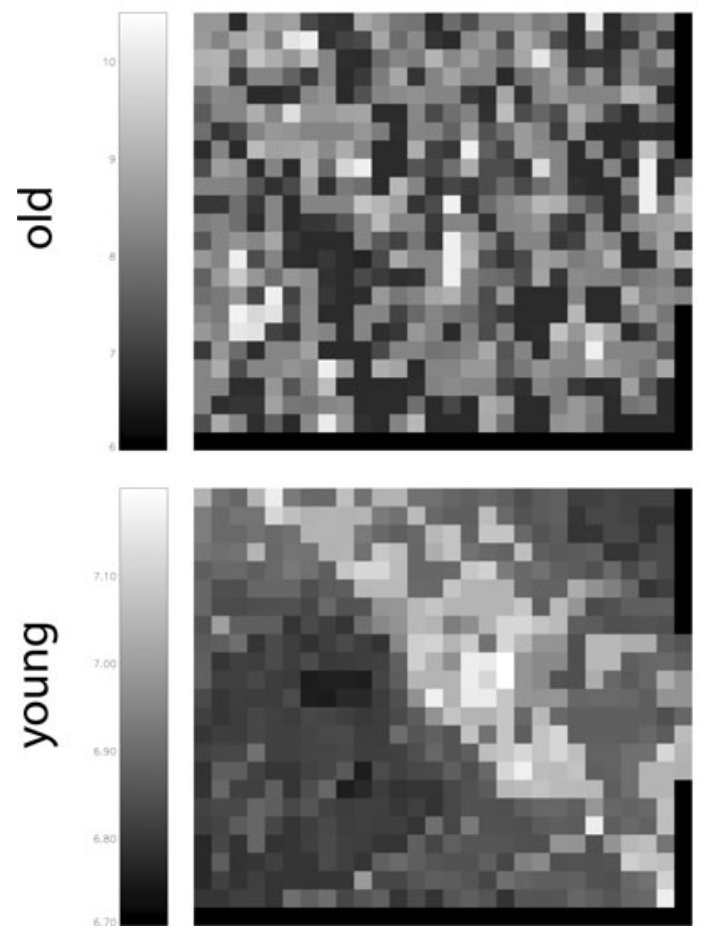

Z
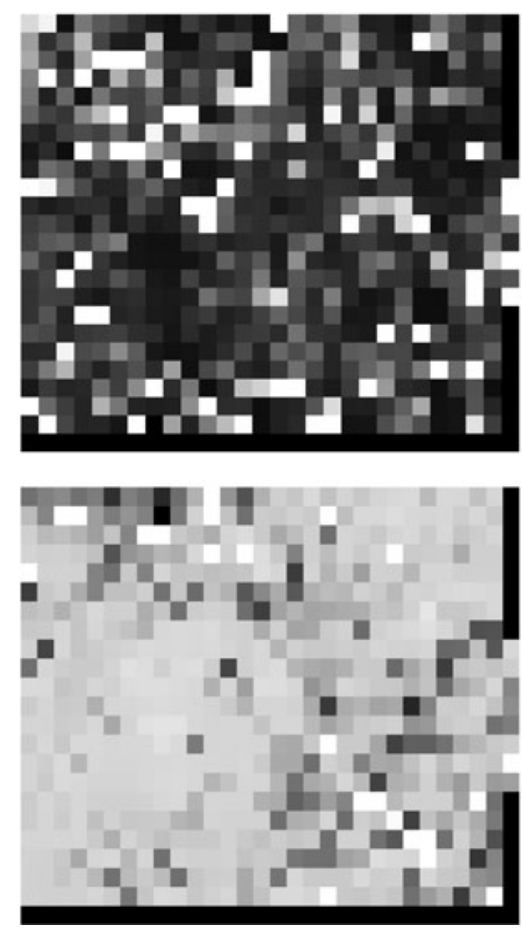
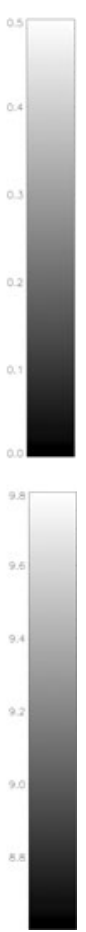

Figure 2. The results for NGC 4900. Top: (a) age and (b) metallicity Z for old stellar populations. Bottom: (c) age and (d) oxygen abundance, $12+\log (O / H)$, for the young ones.

The maps of absorption spectral indices Mg2 and $<\mathrm{Fe}>=(\mathrm{Fe} 5270+\mathrm{Fe} 5335) / 2$ are in the two bottom rows. Mg2 is more dependent on stellar age than $<\mathrm{Fe}>$, which depends strongly on metallicity Z. According to data, $\mathrm{Z}$ is more uniform within each galaxy than the stellar age, which presents strong differences between the emission regions and the absorption ones. In fact, there is a clear gradient between the inner and the outer regions, the last ones being much older that the first ones, at least in NGC 2718, NGC 4385, and NGC 5430. NGC 4900 shows, once again, a peculiar behavior with a young stellar populations located in the bar region, out of center.

\section{Interpretation of data: using evolutionary synthesis models}

The emission lines are interpreted by using the LavalSB (Stb99) code (Dionne \& Robert 2006) to estimate the age of the young stellar populations able to ionize the gas within each region. The absorption spectral indices are interpreted by means of evolutionary synthesis models in high resolution, as the ours, from González-Delgado et al. (2005). In order to estimate age and $\mathrm{Z}$ from spectral indices, we have previously measured them over the SSPs spectra, using the same method we used for our observed spectra. Then, we have performed a Bayesian analysis to select the best age and metallicity.

We show in Fig. 2 the results corresponding to NGC 4900. We see at the top-right panel the stellar metallicity, $\mathrm{Z}$, which is more or less uniform around $[\mathrm{Fe} / \mathrm{H}]=\mathrm{Z}_{\odot} / 3$, with slight quantitative differences at the North-East side, where it is higher: $<[\mathrm{Fe} / \mathrm{H}]>\sim 0.6 \mathrm{Z}_{\odot}$. The stellar age (top-left panel) is of few $100 \mathrm{Myr}$ as mean, and 1 Gyr as maximum, (log age in the range 8-9), with small and not significant variations among regions. 
In the bottom panels, we see the age and oxygen abundance of the last dominating burst. The youngest region with 6-8 Myrs is on the South-East side and the average oxygen abundance is around $12+\log (\mathrm{O} / \mathrm{H})=9.29$, while out of there it is slightly lower and $\log$ age $>7$. Since its shape coincides with the axis of the galaxy bar, this enormous HII region (around 200 right long) may be interpreted as an effect of the bar existence. Bars provoke the inflow of gas proceeding from the disk, and, in consequence, strong star-bursts which, in turn, increases the oxygen abundance.

We have also estimated the stellar masses in each (age,Z) group. On average we have $<140 \times 10^{6} \mathrm{M}_{\odot}$ for each $30 \mathrm{pc}^{2}$ in the old component. The young component has a mass of $<250 \times 10^{3} \mathrm{M}_{\odot}$ in the same surface, with the highest value along the galaxy bar. Thus, the ratio young/old is around $10^{-3}$. We may also get some clues about the star formation rates $(\mathrm{SFR}): \mathrm{SFR}_{\text {past }} \sim 0.014 \mathrm{M}_{\odot} \mathrm{yr}^{-1} \mathrm{kpc}^{-2}$, if we assume that the old stars last at least 1 Gyr to form. The young stellar populations have been created in a time of some Myr, between 15 and $100 \mathrm{Myr}$, and then $\mathrm{SFR}_{\text {present }} \sim 2.510^{-3}-1.510^{-2} \mathrm{M}_{\odot} \mathrm{yr}^{-1} \mathrm{kpc}^{-2}$. This implies that the ratio of past to present star formation rate is around $10-50$.

The center of NGC 4900 host, therefore, many star formation episodes: it suffered a burst in the whole central region $1 \mathrm{Gyr}$ ago what may be associated with the bulge population. Another burst has occurred $\sim 100$ Myr ago, refilling the very central region; then 13-14 Myr ago some small knots of formation took place along the bar structure, with a burst 8 Myr ago at the North-West and an episode 5-6 Myr ago in the South-East.

Can we interpret these data in terms of evolutionary sequences? We might consider two hypotheses: 1) There are only two populations: a bulge with an old stellar population uniform in age and Z, and then the bar provoked a abrupt infall of gas which produces a second younger starburst. In that case the classical method of using SSPs to estimate a mean metallicity and age, as shown before, is valid. 2) If, however, the stars formed in a posterior phase are the consequence of a slow infall of gas (Domínguez-Tenreiro, this volume), which is possible from the differences found in ages along the bar, then the situation changes since a continuous star formation history, $\Psi(t)$, has taken place. When using SSP's, a given function for $\Psi(t)$ is usually assumed in those cases, (e.g. Moorthy \& Holtzman 2006, Ganda et al. 2007), and included it in the equation of deconvolution: $F_{\lambda}(t)=\int_{0}^{t} S_{\lambda}(\tau, Z) \Psi\left(t^{\prime}\right) d t^{\prime}$, (being $\tau=t-t^{\prime}$, and $S_{\lambda}$ the SED of the SSP's). Using this method, maybe the whole information or the correct conclusions are not obtained, since the enrichment history, $\mathrm{Z}(\mathrm{t})$, corresponding to the assumed star formation history is not taken into account. actually $S_{\lambda}(\tau, Z)=S_{\lambda}\left(\tau, Z\left(t^{\prime}\right)\right)$, Z(t) being a function variable in time. In order to face this problem we suggest the use of a set of spectral absorption indices obtained from a chemical evolution model grid (Mollá \& Díaz 2005) as templates, to determine in a consistent way the mean age and metallicity of our regions.

\section{References}

Cardelli, J. A., Clayton, G. C., \& Mathis, J. S. 1989, ApJ 345, 245

Dionne, D. \& Robert, C. 2006, ApJ 641, 252

Ganda, K., et al. 2007, ArXiv e-prints, 706, arXiv:0706.3624

Delgado, R. M., Cerviño, M., Martins, L. P., Leitherer, C., \& Hauschildt, P. H. 2005, MNRAS 357,945

Kewley, L. J. \& Dopita, M. A. 2002, ApJS 142, 35

Mollá, M., \& Díaz, A. I. 2005, MNRAS 358, 521

Moorthy, B. K. \& Holtzman, J. A. 2006, MNRAS 371, 583 